\title{
A class of weighted Hardy type inequalities in $\mathbb{R}^{N}$
}

\section{Anna Canale ${ }^{1}[$}

Received: 25 June 2021 / Revised: 23 July 2021 / Accepted: 28 July 2021

(c) The Author(s) 2021

\section{Abstract}

In the paper we prove the weighted Hardy type inequality

$$
\int_{\mathbb{R}^{N}} V \varphi^{2} \mu(x) d x \leq \int_{\mathbb{R}^{N}}|\nabla \varphi|^{2} \mu(x) d x+K \int_{\mathbb{R}^{N}} \varphi^{2} \mu(x) d x,
$$

for functions $\varphi$ in a weighted Sobolev space $H_{\mu}^{1}$, for a wider class of potentials $V$ than inverse square potentials and for weight functions $\mu$ of a quite general type. The case $\mu=1$ is included. To get the result we introduce a generalized vector field method. The estimates apply to evolution problems with Kolmogorov operators

$$
L u=\Delta u+\frac{\nabla \mu}{\mu} \cdot \nabla u
$$

perturbed by singular potentials.

Keywords Weighted Hardy type inequalities · Kolmogorov operators · Singular potentials · Evolution problems

Mathematics Subject Classification 35K15 · 35K65 - 35B25 · 34G10 - 47D03

\section{Introduction}

The main purpose of the paper is to state a class of inequalities in $\mathbb{R}^{N}$ which generalizes the weighted Hardy inequalities to the case of a more general type of potentials than

The author is member of the Gruppo Nazionale per l'Analisi Matematica, la Probabilitá e le loro Applicazioni of the Istituto Nazionale di Alta Matematica.

$凶 \quad$ Anna Canale

acanale@unisa.it

1 Dipartimento di Ingegneria dell'Informazione ed Elettrica e Matematica Applicata (Diem), Universitá degli Studi di Salerno, Via Giovanni Paolo II, 132, 84084 Fisciano, Salerno, Italy 
inverse square potentials. The method we introduce to get the result is a generalization of the vector field technique used in [19] to prove the classical Hardy inequality. The estimates we prove fit in the framework of the study of boundedness of multiplication operators defined in Sobolev spaces and of the related embedding results.

When $V$ is an inverse square potential, weighted Hardy inequalities have been studied in $[10,17]$, in the case of Gaussian measures, and in $[8,11,12]$ in the setting of more general measures.

In the paper we state the inequality

$$
\int_{\mathbb{R}^{N}} V \varphi^{2} \mu(x) d x \leq \int_{\mathbb{R}^{N}}|\nabla \varphi|^{2} \mu(x) d x+K \int_{\mathbb{R}^{N}} \varphi^{2} \mu(x) d x,
$$

with $\mathrm{K}$ constant, for functions in a weighted Sobolev space $H_{\mu}^{1}$, for a class of potentials $V, 0 \leq V \in L_{l o c}^{1}\left(\mathbb{R}^{N}\right)$, and for weight functions $\mu$ of a quite general type.

The estimate includes the case of the Lebesgue measure and the weighted Hardy inequality with $V$ inverse square potential.

The main reason we are interested in this kind of inequality lies in the applications to the study of Kolmogorov operators

$$
L u=\Delta u+\frac{\nabla \mu}{\mu} \cdot \nabla u,
$$

defined on smooth functions, perturbed by singular potentials, and of the related evolution problems

$$
\text { (P) } \begin{cases}\partial_{t} u(x, t)=L u(x, t)+V(x) u(x, t), & x \in \mathbb{R}^{N}, t>0, \\ u(\cdot, 0)=u_{0} \geq 0, & u_{0} \in L_{\mu}^{2},\end{cases}
$$

where $L_{\mu}^{2}:=L^{2}\left(\mathbb{R}^{N}, d \mu\right)$, with $d \mu(x)=\mu(x) d x$.

In the applications to existence of weak positive solutions to problem $(P)$ we follow Cabré-Martel's approach using the relation between the weak solution of $(P)$ and the bottom of the spectrum of the operator $-(L+V)$

$$
\lambda_{1}(L+V):=\inf _{\varphi \in H_{\mu}^{1} \backslash\{0\}}\left(\frac{\int_{\mathbb{R}^{N}}|\nabla \varphi|^{2} d \mu-\int_{\mathbb{R}^{N}} V \varphi^{2} d \mu}{\int_{\mathbb{R}^{N}} \varphi^{2} d \mu}\right) .
$$

When $\mu=1$ Cabré and Martel in [2] showed that the boundedness of $\lambda_{1}(\Delta+V)$ is a necessary and sufficient condition for the existence of positive exponentially bounded in time solutions to the associated initial value problem. Later in $[8,12,17]$ similar results have been extended to Kolmogorov operators perturbed by inverse square potentials. The proof uses some properties of the operator $L$ and of its corresponding semigroup in $L_{\mu}^{2}\left(\mathbb{R}^{N}\right)$.

The existence of positive solutions to $(P)$ is related to the Hardy type inequality (2). This is due to the relationship between the estimate of the bottom of the spectrum $\lambda_{1}(L+V)$ and the inequality. 
The paper is organized as follows.

In Sect. 2 we present the class of potentials we consider in the paper and give some examples. In Sect. 3 we state, as a first step, an unweighted Hardy type inequality introducing a generalized vector field method and observing the connection with the embedding results. In Sect. 4 we introduce a class of weight functions and state a weighted inequality. Section 5 is devoted to an application to evolution problems.

\section{A class of potentials}

The class of potentials $V$ we consider in unweighted case is of the type

$\left(H_{1}\right) \quad V=V(x) \in L_{l o c}^{1}\left(\mathbb{R}^{N}\right)$ and $0 \leq V \leq V_{f}:=-\frac{\Delta f}{f}$, where the radial functions $f$ fulfil

(H) (i) $f>0, \quad f \in C^{2}\left(\mathbb{R}^{N}\right)$ or $f \in C^{2}\left(\mathbb{R}^{N} \backslash\{0\}\right)$;

(ii) $-\frac{\Delta f}{f} \geq 0$.

Under the condition $f \in C^{2}\left(\mathbb{R}^{N}\right)$ in $\left.H_{2}\right)$, we can integrate by parts in the proof of the inequalities in the next Sections. We need $i i$ ) in $H_{2}$ ) to apply Fatou's lemma in the proof and to obtain nonnegative potentials $V$ on the left-hand side in the inequalities.

The class of radial functions $f$ for $|x|=\rho \in\left[r_{0},+\infty\right.$ [ satisfying $H_{2}$ ) is such that

$$
f^{\prime \prime}+\frac{N-1}{\rho} f^{\prime}=\frac{\left(f^{\prime} \rho^{N-1}\right)^{\prime}}{\rho^{N-1}} \leq 0,
$$

where $f^{\prime}, f^{\prime \prime}$ are the first and the second derivatives with respect to $\rho$, respectively. So the radial functions are such that $f^{\prime} \rho^{N-1}$ is decreasing, from which

$$
f^{\prime}(r) \leq \frac{f^{\prime}\left(r_{0}\right) r_{0}^{N-1}}{r^{N-1}}, \quad r_{0} \leq r .
$$

If we integrate in $\left[r_{0}, r\right], r_{0}>0$, we get

$$
f(r) \leq \frac{c_{1}}{r^{N-2}}+c_{2}
$$

where $c_{1}=-\frac{f^{\prime}\left(r_{0}\right) r_{0}^{N-1}}{N-2}$ and $c_{2}=\frac{f^{\prime}\left(r_{0}\right) r_{0}}{N-2}+f\left(r_{0}\right)$, or, equivalently,

$$
f(r) \leq \frac{c_{3}}{r^{N-2}}-\frac{c_{3}}{r_{0}^{N-2}}+f\left(r_{0}\right),
$$

where $c_{3}=-\frac{f^{\prime}\left(r_{0}\right) r_{0}^{N-1}}{N-2}$, that is the function $f(r)-\frac{c_{3}}{r^{N-2}}$ is decreasing.

This class includes the function

$$
\left.f_{\varepsilon}(x)=\frac{1}{\left(\varepsilon+|x|^{2}\right)^{\frac{\alpha}{2}}}, \quad \varepsilon>0, \quad \alpha \in\right] 0, N-2[,
$$


used to get the Hardy inequality with potential $\left.\left.V=\frac{c}{|x|^{2}}, c \in\right] 0,(N-2)^{2} / 4\right]$ in [11] and, through the vector field method, with $\alpha=2$, in [19] .

An example of function $f \in C^{2}\left(\mathbb{R}^{N} \backslash\{0\}\right)$ verifying $\left.H_{2}\right)$ is represented by $f(x)=$ $\frac{1}{\log ^{\alpha}(1+|x|)}$, for $\left.\left.\alpha \in\right] 0, N-2\right]$, which generates a potential

which behaves like $\frac{c_{\alpha}}{|x|^{2}}$ near to zero.

In the case when $f(x)=\frac{\log (1+|x|)}{|x|^{\alpha}}, \alpha \in[1, N-2]$, the potential

$$
V_{f}=\frac{\alpha(N-2-\alpha)}{|x|^{2}}-\frac{N-1-2 \alpha}{|x|(1+|x|) \log (1+|x|)}+\frac{1}{(1+|x|)^{2} \log (1+|x|)} .
$$

behaves like $\frac{c_{\alpha}}{|x|^{2}}$ when $|x|$ goes to zero if $N \geq 4$ and as $\frac{1}{|x|}$ if $N=3$. If $\alpha \geq \frac{N-1}{2}$, we get

$$
V=\frac{-\alpha^{2}+\alpha(N-2)}{|x|^{2}} \leq V_{f}
$$

Another admissible potential $V$ related to $f$ is

$$
V=\frac{1}{(1+|x|)^{2} \log (1+|x|)} \leq V_{f},
$$

where the singularity in zero is of different type.

In general, under assumptions $H_{2}$ ), the functions $f=f(r)$ solves the linear differential equation for suitable potentials $V_{f}$

$$
f^{\prime \prime}+\frac{N-1}{r} f^{\prime}+f V_{f}=0
$$

or equivalently, by placing $u=\frac{f^{\prime}}{f}$, the Riccati equation

$$
u^{\prime}=-u^{2}-\frac{N-1}{r} u-V_{f}
$$

In a forthcoming paper we study examples of functions $f$ to get improved Hardy inequality in bounded sets in $\mathbb{R}^{N}$.

\section{First step: unweighted Hardy type inequalities}

The estimate in the next Theorem concerns the case of Lebesgue measure.

Theorem 1 Under the condition $\mathrm{H}_{1}$ ) and $\mathrm{H}_{2}$ ), we get the estimate

$$
\int_{\mathbb{R}^{N}} V \varphi^{2} d x \leq \int_{\mathbb{R}^{N}}|\nabla \varphi|^{2} d x
$$

for any functions $\varphi \in H^{1}\left(\mathbb{R}^{N}\right)$. 
Proof By density, it is enough to prove (5) for every $\varphi \in C_{c}^{\infty}\left(\mathbb{R}^{N}\right)$.

As a first step we suppose $f \in C^{2}\left(\mathbb{R}^{N}\right)$ and prove that it holds

$$
-\int_{\mathbb{R}^{N}} \frac{\Delta f}{f} \varphi^{2} d x \leq \int_{\mathbb{R}^{N}}|\nabla \varphi|^{2} d x .
$$

To this aim we introduce the vectorial function

$$
F=-\nabla \log f
$$

The starting point is the following integral

$$
\begin{aligned}
\int_{\mathbb{R}^{N}} \operatorname{div} F \varphi^{2} d x & =-\int_{\mathbb{R}^{N}} \Delta \log f \varphi^{2} d x \\
& =-\int_{\mathbb{R}^{N}} \frac{\Delta f}{f} \varphi^{2} d x+\int_{\mathbb{R}^{N}}\left|\frac{\nabla f}{f}\right|^{2} \varphi^{2} d x .
\end{aligned}
$$

The next step is to estimate the integral on the left-hand side in (7) from above. We integrate by parts taking into account that $F_{j}, \frac{\partial F_{j}}{\partial x_{j}} \in L_{l o c}^{1}\left(\mathbb{R}^{N}\right)$ where $F_{j}=-\frac{1}{f} \frac{\partial f}{\partial x_{j}}$. Using Hölder's and Young's inequalities we get

$$
\begin{aligned}
\int_{\mathbb{R}^{N}} \operatorname{div} F \varphi^{2} d x= & -2 \int_{\mathbb{R}^{N}} \varphi F \cdot \nabla \varphi d x \\
& \leq 2\left(\int_{\mathbb{R}^{N}}|\nabla \varphi|^{2} d x\right)^{\frac{1}{2}}\left(\int_{\mathbb{R}^{N}}\left|\frac{\nabla f}{f}\right|^{2} \varphi^{2} d x\right)^{\frac{1}{2}} \\
& \leq \int_{\mathbb{R}^{N}}|\nabla \varphi|^{2} d x+\int_{\mathbb{R}^{N}}\left|\frac{\nabla f}{f}\right|^{2} \varphi^{2} d x
\end{aligned}
$$

Putting together (7) and (8) we have

$$
\begin{aligned}
& -\int_{\mathbb{R}^{N}} \frac{\Delta f}{f} \varphi^{2} d x+\int_{\mathbb{R}^{N}}\left|\frac{\nabla f}{f}\right|^{2} \varphi^{2} d x \\
& \leq \int_{\mathbb{R}^{N}}|\nabla \varphi|^{2} d x+\int_{\mathbb{R}^{N}}\left|\frac{\nabla f}{f}\right|^{2} \varphi^{2} d x
\end{aligned}
$$

and, then, (6).

If $f \in C^{2}\left(\mathbb{R}^{N} \backslash\{0\}\right)$, reviewing the radial function $f$ as $f=f\left(|x|^{2}\right)$, we remove the singularity introducing the corresponding function $f_{\varepsilon}, \varepsilon>0$, as $f_{\varepsilon}=$ $f\left(\varepsilon+|x|^{2}\right) \in C^{2}\left(\mathbb{R}^{N}\right)$. This is why the gradient of $|x|^{2}$ is not singular in zero. So we get $\lim _{\varepsilon \rightarrow 0} f_{\varepsilon}(x)=f(x)$ and $\Delta f_{\varepsilon} \rightarrow \Delta f$. Then we reason as above to get

$$
-\int_{\mathbb{R}^{N}} \frac{\Delta f_{\varepsilon}}{f_{\varepsilon}} \varphi^{2} d x \leq \int_{\mathbb{R}^{N}}|\nabla \varphi|^{2} d x .
$$


Letting $\varepsilon \rightarrow 0$ on the left-hand side in (10), by Fatou's lemma and $H_{1}$ ), we deduce the inequality (5).

Remark 1 We obtain the classical Hardy inequality if $f(x)=\frac{1}{|x|^{\alpha}}$, for $\left.\alpha \in\right] 0, N-2[$. We remove the singularity in zero introducing $f_{\varepsilon}(x)=f\left(\varepsilon+|x|^{2}\right)=\frac{1}{\left(\varepsilon+|x|^{2}\right)^{\frac{\alpha}{2}}}$ (cf. (4)) which generates the vectorial function $F(x)=\alpha \frac{x}{\varepsilon+|x|^{2}}$. The potential is $V_{f}=\frac{c}{|x|^{2}}, c=\alpha(N-2-\alpha)$. The maximum value of the constant $c$ is $c_{o}=\frac{(N-2)^{2}}{4}$ attained for $\alpha=\frac{N-2}{2}$. In general we cannot state the optimality of the inequality (5).

Furthermore we remark that the method we use in the proof of the Theorem 1, in the case $f_{\varepsilon}(x)=\frac{1}{\varepsilon+|x|^{2}}$, results to be the vector field method used in [19] to prove the Hardy inequality.

As a consequence of Theorem 1 we deduce the boundedness of the multiplication operator

$$
\varphi \rightarrow V^{\frac{1}{2}} \varphi
$$

defined in the Sobolev space $H^{1}\left(\mathbb{R}^{N}\right)$ and which takes values in $L^{2}\left(\mathbb{R}^{N}\right)$

$$
\left\|V^{\frac{1}{2}} \varphi\right\|_{L^{2}\left(\mathbb{R}^{N}\right)} \leq c\|\varphi\|_{H^{1}\left(\mathbb{R}^{N}\right)},
$$

where $c$ is a constant independent of $V$ and $\varphi$, and, then, we get an embedding result.

For $L^{p}$ estimates for multiplication operators and embedding results of this type with some applications to elliptic equations see $[3-7,16]$.

\section{Weighted Hardy type inequalities}

Now we consider the Hardy type inequality in the weighted case. Let $\mu \geq 0$ be a weight function on $\mathbb{R}^{N}$. We define the weighted Sobolev space $H_{\mu}^{1}=H^{1}\left(\mathbb{R}^{N}, \mu(x) d x\right)$ as the space of functions in $L_{\mu}^{2}:=L^{2}\left(\mathbb{R}^{N}, \mu(x) d x\right)$ whose weak derivatives belong to $L_{\mu}^{2}$.

The conditions on $\mu$ which we need are the following.

$\left(H_{3}\right)$ (i) $\sqrt{\mu} \in H_{l o c}^{1}\left(\mathbb{R}^{N}\right)$;

(ii) $\mu^{-1} \in L_{\text {loc }}^{1}\left(\mathbb{R}^{N}\right)$;

(iii) there exist constants $K_{1}, K_{2} \in \mathbb{R}$ such that under the hypothesis $H_{2}$ ) on $f$ it holds

$$
\nabla \log f \cdot \nabla \mu \leq\left(K_{1}+K_{2} \frac{x}{|x|^{2}} \cdot \frac{\nabla f}{f}\right) \mu
$$

with $K_{2}$ so that

$$
-\frac{\Delta f}{f}-K_{2} \frac{x}{|x|^{2}} \cdot \frac{\nabla f}{f}=-\frac{f^{\prime \prime}}{f}-\frac{\left(N+K_{2}-1\right)}{r} \frac{f^{\prime}}{f} \geq 0 .
$$


Let us observe that under the assumptions $(i)$ and $(i i)$ in the hypothesis $H_{3}$ ) the space $C_{c}^{\infty}\left(\mathbb{R}^{N}\right)$ is dense in $H_{\mu}^{1}$ (see e.g. [20]). So we can regard $H_{\mu}^{1}$ as the completion of $C_{c}^{\infty}\left(\mathbb{R}^{N}\right)$ with respect to the Sobolev norm

$$
\|\cdot\|_{H_{\mu}^{1}}^{2}:=\|\cdot\|_{L_{\mu}^{2}}^{2}+\|\nabla \cdot\|_{L_{\mu}^{2}}^{2} \cdot
$$

The condition (13) is the same as $i i$ ) in $H_{2}$ ) with $N+K_{2}$ in place of $N$.

The condition (12) states a relation between $f$ and $\mu$ and can be rewritten as follows

$$
\frac{f^{\prime}}{f}\left(\frac{\mu^{\prime}}{\mu}-\frac{K_{2}}{r}\right) \leq K_{1}, \quad r=|x| .
$$

For $f$ fixed, it is a condition for $\mu$. For example if $f$ is as in (4), good weight functions to get weighted Hardy inequalities are the functions

$$
\mu(x)=\frac{1}{|x|^{\gamma}} e^{-\delta|x|^{m}}, \quad \delta \geq 0, \quad \gamma<N-2,
$$

for suitable values of $\gamma$ and $m$ (see [11]). Conversely, for $\mu$ fixed, (14) represents a condition on $f$. If, for example, $\mu(x)=e^{-\delta|x|^{2}}$, a Gaussian function, $K_{2}>0$, starting with (14) and integrating in $\left[r_{0}, r\right], r_{0}>0$, we get the necessary condition

$$
f(r) \geq f\left(r_{0}\right)\left(\frac{2 \delta r_{0}^{2}+K_{2}}{2 \delta r^{2}+K_{2}}\right)^{\frac{K_{1}}{4 \delta}} .
$$

The assumption on the potential $V$ in the weighted case is the following

$\left.H_{4}\right) \quad V=V(x) \in L_{l o c}^{1}\left(\mathbb{R}^{N}\right)$ and

$$
0 \leq V \leq V_{f}:=-\frac{\Delta f}{f}-K_{2} \frac{x}{|x|^{2}} \cdot \frac{\nabla f}{f} .
$$

The next result is an inequality of Hardy type with weight. To obtain the result we introduce a suitable vectorial function $F$ and use the method introduced in Sect. 2 in the case $\mu=1$.

Theorem 2 Under the conditions $(i)$ in $\left(H_{2}\right),\left(H_{3}\right)$ and $\left(H_{4}\right)$, the following inequality with potential $V$ holds

$$
\int_{\mathbb{R}^{N}} V \varphi^{2} d \mu \leq \int_{\mathbb{R}^{N}}|\nabla \varphi|^{2} d \mu+K_{1} \int_{\mathbb{R}^{N}} \varphi^{2} d \mu,
$$

for any functions $\varphi \in H_{\mu}^{1}$.

Proof As in the Theorem 1 it is enough to prove (16) for any $\varphi \in C_{c}^{\infty}\left(\mathbb{R}^{N}\right)$. As in the previous proof we suppose $f \in C^{2}\left(\mathbb{R}^{N}\right)$ at first. 
We introduce the function

$$
F=-\mu \nabla \log f
$$

The divergence of the vector field assumes the form

$$
\operatorname{div} F=-\mu \Delta \log f-\nabla \log f \cdot \nabla \mu=-\frac{\Delta f}{f} \mu+\left|\frac{\nabla f}{f}\right|^{2} \mu-\frac{\nabla f}{f} \cdot \nabla \mu
$$

So, by hypothesis $(i i i)$ in $\left(H_{3}\right)$, we obtain from below

$$
\begin{aligned}
\int_{\mathbb{R}^{N}} \operatorname{div} F \varphi^{2} d x=- & \int_{\mathbb{R}^{N}} \frac{\Delta f}{f} \varphi^{2} d \mu+\int_{\mathbb{R}^{N}}\left|\frac{\nabla f}{f}\right|^{2} \varphi^{2} d \mu \\
& -\int_{\mathbb{R}^{N}} \frac{\nabla f}{f} \cdot \frac{\nabla \mu}{\mu} \varphi^{2} d \mu \geq \int_{\mathbb{R}^{N}} \frac{\Delta f}{f} \varphi^{2} d \mu \\
& +\int_{\mathbb{R}^{N}}\left|\frac{\nabla f}{f}\right|^{2} \varphi^{2} d \mu \\
& -K_{1} \int_{\mathbb{R}^{N}} \varphi^{2} d \mu-K_{2} \int_{\mathbb{R}^{N}} \frac{x}{|x|^{2}} \cdot \frac{\nabla f}{f} \varphi^{2} d \mu .
\end{aligned}
$$

To estimate the integral on the left-hand side in (17) from above, reasoning as in the proof of Theorem 1 to get (8), we integrate by parts.

To this aim we need to $F_{j}, \frac{\partial F_{j}}{\partial x_{j}} \in L_{l o c}^{1}\left(\mathbb{R}^{N}\right)$ where $F_{j}$, for $j=1, \ldots n$, is the $\mathrm{j}$-th component of $F$. For the partial derivatives of $F_{j}$, it is sufficient that $-\frac{1}{f} \frac{\partial f}{\partial x_{j}} \sqrt{\mu} \in$ $L_{l o c}^{2}\left(\mathbb{R}^{N}\right)$ and $\frac{1}{f} \frac{\partial^{2} f}{\partial x_{j}^{2}} \mu \in L_{l o c}^{1}\left(\mathbb{R}^{N}\right)$. In particular, for any $K$ compact set in $\mathbb{R}^{N}, F_{j}$ belongs to $L_{\text {loc }}^{1}\left(\mathbb{R}^{N}\right)$ since $\mu \in L_{\text {loc }}^{1}\left(\mathbb{R}^{N}\right)$ by $(i)$ in $\left(H_{3}\right)$.

On the other hand

$$
\begin{gathered}
\int_{K}\left|\frac{\partial F_{j}}{\partial x_{j}}\right| d x \leq \int_{K}\left|\frac{1}{f} \frac{\partial f}{\partial x_{j}}\right|^{2} \mu(x) d x+\int_{K}\left|-\frac{1}{f} \frac{\partial^{2} f}{\partial x_{j}^{2}}\right| \mu(x) d x \\
+\int_{K}\left|-\frac{1}{f} \frac{\partial f}{\partial x_{j}} \frac{\partial \mu}{\partial x_{j}}\right| d x .
\end{gathered}
$$

The last term on the right-hand side in (18) can be estimated using Hölder's inequality

$$
\begin{aligned}
\int_{K}\left|-\frac{1}{f} \frac{\partial f}{\partial x_{j}} \frac{\partial \mu}{\partial x_{j}}\right| d x & \leq\left(\int_{K}\left|-\frac{1}{f} \frac{\partial f}{\partial x_{j}}\right|^{2} \mu(x) d x\right)^{\frac{1}{2}}\left(\int_{K}\left|\frac{1}{\sqrt{\mu}} \frac{\partial \mu}{\partial x_{j}}\right|^{2} d x\right)^{\frac{1}{2}} \\
& \leq 4\left(\int_{K}\left|-\frac{1}{f} \frac{\partial f}{\partial x_{j}}\right|^{2} d \mu\right)^{\frac{1}{2}}\left(\int_{K}|\nabla \sqrt{\mu}|^{2} d x\right)^{\frac{1}{2}} .
\end{aligned}
$$


Then $\frac{\partial F_{j}}{\partial x_{j}}$ is locally integrable by $(i)$ in $\left(H_{3}\right)$. In analogy to $(8)$ we get

$$
\begin{aligned}
\int_{\mathbb{R}^{N}} \operatorname{div} F \varphi^{2} d x= & -2 \int_{\mathbb{R}^{N}} \varphi F \cdot \nabla \varphi d x \\
& \leq \int_{\mathbb{R}^{N}}|\nabla \varphi|^{2} d \mu+\int_{\mathbb{R}^{N}}\left|-\frac{\nabla f}{f}\right|^{2} \varphi^{2} d \mu .
\end{aligned}
$$

From (17) and (19) we deduce that

$$
\begin{aligned}
& -\int_{\mathbb{R}^{N}} \frac{\Delta f}{f} \varphi^{2} d \mu-K_{2} \int_{\mathbb{R}^{N}}\left(\frac{x}{|x|^{2}} \cdot \frac{\nabla f}{f}\right) \varphi^{2} d \mu \\
& \leq \int_{\mathbb{R}^{N}}|\nabla \varphi|^{2} d \mu+K_{1} \int_{\mathbb{R}^{N}} \varphi^{2} d \mu .
\end{aligned}
$$

If $f \in C^{2}\left(\mathbb{R}^{N} \backslash\{0\}\right)$ we argue as in the proof of Theorem 1 to avoid the singularity by means the introduction of functions $f_{\varepsilon} \in C^{2}\left(\mathbb{R}^{N}\right)$ which approximate $f$ getting

$$
\begin{aligned}
& -\int_{\mathbb{R}^{N}} \frac{\Delta f_{\varepsilon}}{f_{\varepsilon}} \varphi^{2} d \mu-K_{2} \int_{\mathbb{R}^{N}} \frac{x}{|x|^{2}} \cdot \frac{\nabla f_{\varepsilon}}{f_{\varepsilon}} \varphi^{2} d \mu \\
& \leq \int_{\mathbb{R}^{N}}|\nabla \varphi|^{2} d \mu+K_{1} \int_{\mathbb{R}^{N}} \varphi^{2} d \mu .
\end{aligned}
$$

The condition (13) in $\left(H_{3}\right)$ allow us to apply Fatou's lemma. So, letting $\varepsilon \rightarrow 0$ on the left-hand side in (21) we deduce (20) and, so, the inequality (16) taking into account (15).

A weighted Hardy inequality was stated in [11] with $f$ as in (4), which generates the potential $V=\frac{c}{|x|^{2}}$, with $\left.\left.c \in\right] 0,\left(N+K_{2}-2\right)^{2} / 4\right]$, using a different method. We observe that the Theorem 2 includes the case of inverse square potentials.

Remark 2 In an alterative way we can suppose

$$
-\frac{1}{f} \frac{\partial f}{\partial x_{j}} \sqrt{\mu} \in L_{l o c}^{2}\left(\mathbb{R}^{N}\right), \quad \frac{1}{f} \frac{\partial^{2} f}{\partial x_{j}^{2}} \mu \in L_{l o c}^{1}\left(\mathbb{R}^{N}\right)
$$

in place of hypothesis $(i)$ in $\left(H_{2}\right)$. The proof of the Theorem 2 would be slightly different with more attention to show local integrability and to apply integration by parts.

To conclude, we observe that from Theorem 2 we get the following estimate for the multiplication operator (11)

$$
\left\|V^{\frac{1}{2}} \varphi\right\|_{L_{\mu}^{2}} \leq c\|\varphi\|_{H_{\mu}^{1}}
$$




\section{An application to evolution problems}

The purpose is to show an application of Hardy inequalities to evolution problems of the type

$$
(P) \begin{cases}\partial_{t} u(x, t)=L u(x, t)+V(x) u(x, t), & x \in \mathbb{R}^{N}, t>0, \\ u(\cdot, 0)=u_{0} \geq 0, & u_{0} \in L_{\mu}^{2},\end{cases}
$$

where $L+V$ is the Kolmogorov operator

$$
L u=\Delta u+\frac{\nabla \mu}{\mu} \cdot \nabla u,
$$

on smooth functions, perturbed by a potential $V$ which fulfils condition $H_{4}$ ). The weight function $\mu$ in the drift term belongs to $C_{l o c}^{1, \lambda}\left(\mathbb{R}^{N} \backslash\{0\}\right)$.

In particular we state an existence result for weak solutions to the initial value problem $(P)$ if a Hardy type inequality holds.

We say that $u$ is a weak solution to $(P)$ if, for each $T, R>0$, we have

$$
u \in C\left([0, T], L_{\mu}^{2}\right), \quad V u \in L^{1}\left(B_{R} \times(0, T), d \mu d t\right)
$$

and

$$
\int_{0}^{T} \int_{\mathbb{R}^{N}} u\left(-\partial_{t} \phi-L \phi\right) d \mu d t-\int_{\mathbb{R}^{N}} u_{0} \phi(\cdot, 0) d \mu=\int_{0}^{T} \int_{\mathbb{R}^{N}} V u \phi d \mu d t
$$

for all $\phi \in W_{2}^{2,1}\left(\mathbb{R}^{N} \times[0, T]\right)$ having compact support with $\phi(\cdot, T)=0$, where $B_{R}$ denotes the open ball of $\mathbb{R}^{N}$ of radius $R$ centered at 0 . For any $\Omega \subset \mathbb{R}^{N}, W_{2}^{2,1}(\Omega \times$ $(0, T))$ is the parabolic Sobolev space of the functions $u \in L^{2}(\Omega \times(0, T))$ having weak space derivatives $D_{x}^{\alpha} u \in L^{2}(\Omega \times(0, T))$ for $|\alpha| \leq 2$ and weak time derivative $\partial_{t} u \in L^{2}(\Omega \times(0, T))$ equipped with the norm

$$
\begin{aligned}
\|u\|_{W_{2}^{2,1}(\Omega \times(0, T))}:= & \left(\|u\|_{L^{2}(\Omega \times(0, T))}^{2}+\left\|\partial_{t} u\right\|_{L^{2}(\Omega \times(0, T))}^{2}\right. \\
& \left.+\sum_{1 \leq|\alpha| \leq 2}\left\|D^{\alpha} u\right\|_{L^{2}(\Omega \times(0, T))}^{2}\right)^{\frac{1}{2}} .
\end{aligned}
$$

In the standard setting one considers $\mu \in C_{l o c}^{1, \lambda}\left(\mathbb{R}^{N}\right)$ for some $\lambda \in(0,1)$ and $\mu>0$ for any $x \in \mathbb{R}^{N}$. In this case it is known that the operator $L$ with domain

$$
D_{\text {max }}(L)=\left\{u \in C_{b}\left(\mathbb{R}^{N}\right) \cap W_{\text {loc }}^{2, p}\left(\mathbb{R}^{N}\right) \text { for all } 1<p<\infty, L u \in C_{b}\left(\mathbb{R}^{N}\right)\right\}
$$

is the weak generator of a not necessarily $C_{0}$-semigroup in $C_{b}\left(\mathbb{R}^{N}\right)$. Since $\int_{\mathbb{R}^{N}} L u d \mu=0$ for any $u \in C_{c}^{\infty}\left(\mathbb{R}^{N}\right)$, then $d \mu=\mu(x) d x$ is the invariant measure for this semigroup in $C_{b}\left(\mathbb{R}^{N}\right)$. So we can extend it to a positivity preserving and 
analytic $C_{0}$-semigroup $\{T(t)\}_{t \geq 0}$ on $L_{\mu}^{2}$, whose generator is still denoted by $L$ (see [18]).

When the assumptions on $\mu$ allow degeneracy at one point, we require the following conditions to get that $L$ generates a semigroup:

$\left(H_{5}\right) \quad \mu \in C_{l o c}^{1, \lambda}\left(\mathbb{R}^{N} \backslash\{0\}\right), \lambda \in(0,1), \mu \in H_{l o c}^{1}\left(\mathbb{R}^{N}\right), \frac{\nabla \mu}{\mu} \in L_{l o c}^{r}\left(\mathbb{R}^{N}\right)$ for some $r>N$, and $\inf _{x \in K} \mu(x)>0$ for any compact set $K \subset \mathbb{R}^{N}$.

We remark that the condition $\left(H_{5}\right)$ implies $(i)$ in $\left(H_{3}\right)$. Indeed if $\mu \in H_{l o c}^{1}\left(\mathbb{R}^{N}\right)$ then $\mu \in L_{l o c}^{1}\left(\mathbb{R}^{N}\right)$ and $\nabla \mu \in L_{l o c}^{2}\left(\mathbb{R}^{N}\right)$. Moreover $\frac{\nabla \mu}{\mu} \in L_{l o c}^{2}\left(\mathbb{R}^{N}\right)$ since $r>2$. So we get

$$
\int_{K}|\nabla \sqrt{\mu}|^{2} d x=\frac{1}{4} \int_{K} \frac{|\nabla \mu|^{2}}{\mu} d x \leq \frac{1}{4}\left(\int_{K}\left|\frac{\nabla \mu}{\mu}\right|^{2} d x\right)^{\frac{1}{2}}\left(\int_{K}|\nabla \mu|^{2} d x\right)^{\frac{1}{2}} .
$$

An example of weight function satisfying $\left(H_{5}\right)$ is $\mu=e^{-\delta|x|^{m}}, \delta, m>0$.

Under assumption $\left(H_{5}\right)$, by [1, Corollary 3.7] we have that the closure of $\left(L, C_{c}^{\infty}\left(\mathbb{R}^{N}\right)\right)$ on $L_{\mu}^{2}$ generates a strongly continuous and analytic Markov semigroup $\{T(t)\}_{t \geq 0}$ on $L_{\mu}^{2}$.

We remark that in the applications to evolution problems we need $C_{0}$-semigroup generation results. Operators of a more general type, perturbed by potentials with different singularities, for which the generation of semigroups was stated, have been studied in [13-15] when $\mu=1$ and in [9] in weighted spaces.

We define the bottom of the spectrum of $-(L+V)$ as follows

$$
\lambda_{1}(L+V):=\inf _{\varphi \in H_{\mu}^{1} \backslash\{0\}}\left(\frac{\int_{\mathbb{R}^{N}}|\nabla \varphi|^{2} d \mu-\int_{\mathbb{R}^{N}} V \varphi^{2} d \mu}{\int_{\mathbb{R}^{N}} \varphi^{2} d \mu}\right) .
$$

In the case of $\mu \in C_{l o c}^{1, \lambda}\left(\mathbb{R}^{N}\right)$ the authors in [17] extended the existence results in [2] to the Kolmogorov operators.

The following result stated in [8], assuming for $\mu$ the condition $\left(H_{5}\right)$, shows that the condition $\lambda_{1}(L+V)>-\infty$ is a sufficient condition for the existence of an exponentially bounded positive weak solution of $(P)$. We include the condition $(\mathrm{ii})$ in $\left(H_{3}\right)$ to get the density result.

Theorem 3 Let $0 \leq V(x) \in L_{\text {loc }}^{1}\left(\mathbb{R}^{N}\right)$. Assume that the weight function $\mu$ satisfies (ii) in $\left(H_{3}\right)$ and $\left(H_{5}\right)$. Then, if $\lambda_{1}(L+V)>-\infty$ there exists a positive weak solution $u \in C\left([0, \infty), L_{\mu}^{2}\right)$ of $(P)$ satisfying the estimate

$$
\|u(t)\|_{L_{\mu}^{2}} \leq M e^{\omega t}\left\|u_{0}\right\|_{L_{\mu}^{2}}, \quad t \geq 0
$$

for some constants $M \geq 1$ and $\omega \in \mathbb{R}$.

In the next Theorem we state an existence result for weak solutions to the problem $(P)$ associated to the Kolmogorov operators with weights which are not necessarily $(1, \lambda)$-Hölder continuous in the whole space. 
Theorem 4 Assume hypotheses $(i)$ in $\left(H_{2}\right)$ and $\left(H_{4}\right)$ for the potential $V$ and the conditions ( $\mathrm{ii})$, (iii) in $\left(\mathrm{H}_{3}\right)$ and $\left(\mathrm{H}_{5}\right)$ on $\mu$. Then there exists a positive weak solution $u \in C\left([0, \infty), L_{\mu}^{2}\right)$ of $(P)$ satisfying

$$
\|u(t)\|_{L_{\mu}^{2}} \leq M e^{\omega t}\left\|u_{0}\right\|_{L_{\mu}^{2}}, \quad t \geq 0
$$

for some constants $M \geq 1$ and $\omega \in \mathbb{R}$.

Proof Under the hypotheses of the Theorem the inequality (16) holds. The result is a consequence of the Theorem 3 and of the inequality (16) taking into account that the weighted Hardy type inequality implies that $\lambda_{1}(L+V)>-\infty$.

We observe that, in the case of Schrödinger operators, we relay on the result in [2] and on the Hardy type inequality (5) to get the following result.

Theorem 5 Assume hypotheses $\left(H_{1}\right)$ and $\left(H_{2}\right)$. Then there exists a positive weak solution $u \in C\left([0, \infty), L^{2}\left(\mathbb{R}^{N}\right)\right.$ of $(P)$ with $L=\Delta$ and $\mu=1$, satisfying

$$
\|u(t)\|_{L^{2}\left(\mathbb{R}^{N}\right)} \leq M e^{\omega t}\left\|u_{0}\right\|_{L^{2}\left(\mathbb{R}^{N}\right)}, \quad t \geq 0
$$

for some constants $M \geq 1$ and $\omega \in \mathbb{R}$.

Funding Open access funding provided by Università degli Studi di Salerno within the CRUI-CARE Agreement.

\section{Declarations}

Conflict of interest The author declare that they have no conflict of interest.

Open Access This article is licensed under a Creative Commons Attribution 4.0 International License, which permits use, sharing, adaptation, distribution and reproduction in any medium or format, as long as you give appropriate credit to the original author(s) and the source, provide a link to the Creative Commons licence, and indicate if changes were made. The images or other third party material in this article are included in the article's Creative Commons licence, unless indicated otherwise in a credit line to the material. If material is not included in the article's Creative Commons licence and your intended use is not permitted by statutory regulation or exceeds the permitted use, you will need to obtain permission directly from the copyright holder. To view a copy of this licence, visit http://creativecommons.org/licenses/by/4.0/.

\section{References}

1. Albanese, A., Lorenzi, L., Mangino, E.: $L^{p}$-uniqueness for elliptic operators with unbounded coefficients in $\mathbb{R}^{N}$. J. Funct. Anal. 256(4), 1238-1257 (2009). https://doi.org/10.1016/j.jfa.2008.07.022

2. Cabré, X., Martel, Y.: Existence versus explosion instantanée pour des equations de la chaleur lineáires avec potentiel singulier. C. R. Acad. Sci. Paris 329(11), 973-978 (1999). https://doi.org/10.1016/ S0764-4442(00)88588-2

3. Canale, A.: A priori bounds in weighted spaces. J. Math. Anal. Appl. 287, 101-117 (2003). https:// doi.org/10.1016/S0022-247X(03)00534-1

4. Canale, A.: On some results in weighted spaces under Cordes type conditions. J. Interdiscip. Math. 10, 245-261 (2007). https://doi.org/10.1080/09720502.2007.10700490 
5. Canale, A.: $L^{\infty}$ estimates for variational solutions of boundary value problems in unbounded domains. J. Interdiscip. Math. 11, 127-139 (2008). https://doi.org/10.1080/09720502.2008.10700547

6. Canale, A.: Bounds in spaces of Morrey under Chicco type conditions. Math. Inequal. Appl. 12, 265-278 (2009). https://doi.org/10.7153/mia-12-22

7. Canale, A.: An embedding result. J. Interdiscip. Math. 17, 199-206 (2014). https://doi.org/10.1080/ 09720502.2014 .881142

8. Canale, A., Gregorio, F., Rhandi, A., Tacelli, C.: Weighted Hardys inequalities and Kolmogorov-type operators. Appl. Anal. 98(7), 1236-1254 (2019). https://doi.org/10.1080/00036811.2017.1419200

9. Canale, A., Mininni, R.M., Rhandi, A.: Analytic approach to solve a degenerate parabolic PDE for the Heston model. Math. Meth. Appl. Sci. 40, 4982-4992 (2017). https://doi.org/10.1002/mma.4363

10. Canale, A., Pappalardo, F.: Weighted Hardy inequalities and Ornstein-Uhlenbeck type operators perturbed by multipolar inverse square potentials. J. Math. Anal. Appl. 463, 895-909 (2018). https://doi. org/10.1016/j.jmaa.2018.03.059

11. Canale, A., Pappalardo, F., Tarantino, C.: A class of weighted Hardy inequalities and applications to evolution problems. Ann. Mat. Pura Appl. 199, 1171-1181 (2020). https://doi.org/10.1007/s10231019-00916-y

12. Canale, A., Pappalardo, F., Tarantino, C.: Weighted multipolar Hardy inequalities and evolution problems with Kolmogorov operators perturbed by singular potentials. Commun. Pure Appl. Anal. 20, 405-425 (2021). https://doi.org/10.3934/cpaa.2020274

13. Canale, A., Rhandi, A., Tacelli, C.: Schrödinger type operators with unbounded diffusion and potential terms. Ann. Sc. Norm. Super. Pisa Cl. Sci. Serie V 16, 581-601 (2016). https://doi.org/10.2422/20362145.201409-007

14. Canale, A., Rhandi, A., Tacelli, C.: Kernel estimates for Schrödinger type operators with unbounded diffusion and potential terms. Z. Anal. Anwend. 36, 377-392 (2017). https://doi.org/10.4171/ZAA/ 1593

15. Canale, A., Tacelli, C.: Kernel estimates for a Schrödinger type operator. Riv. Mat. Univ. Parma 7, 341-350 (2016)

16. Canale, A., Tarantino, C.: Morrey type spaces and multiplication operators in Sobolev spaces, arXiv: 1412.6778v1, (2014)

17. Goldstein, G.R., Goldstein, J.A., Rhandi, A.: Weighted Hardys inequality and the Kolmogorov equation perturbed by an inverse-square potential. Appl. Anal. 91(11), 2057-2071 (2012). https://doi.org/10. 1080/00036811.2011.587809

18. Lorenzi, L., Bertoldi, M.: Analytical Methods for Markov Semigroups, Pure and Applied Mathematics 283. CRC Press, Boca Raton (2006)

19. Mitidieri, E.: A simple approach to Hardy inequalities. Math. Notes 67(4), 479-486 (2000). https:// doi.org/10.1007/BF02676404

20. Tölle, J.M.: Uniqueness of weighted Sobolev spaces with weakly differentiable weights. J. Funct. Anal. 263, 3195-3223 (2012). https://doi.org/10.1016/j.jfa.2012.08.002

Publisher's Note Springer Nature remains neutral with regard to jurisdictional claims in published maps and institutional affiliations. 